\title{
Determination of urine ionic composition with potentiometric multisensor system
}

\author{
Irina Yaroshenko ${ }^{\mathrm{a}, \mathrm{b}, \mathrm{c}}$, Dmitry Kirsanov ${ }^{\mathrm{a}, \mathrm{c}, *}$, Lyudmila Kartsova ${ }^{\mathrm{a}}$, Alla Sidorova ${ }^{\mathrm{b}}$, \\ Irina Borisova ${ }^{\mathrm{b}}$, Andrey Legin ${ }^{\mathrm{a}, \mathrm{c}}$ \\ ${ }^{a}$ Chemistry Department, St. Petersburg State University, Universitetskaya nab. 7/9, Mendeleev Center, 199034 St. Petersburg, Russia \\ b Bioanalytical Laboratory CSU “Analytical Spectrometry", St. Petersburg State Polytechnical University, Box 27, Gzhatskaya Street 27, \\ 198220 St. Petersburg, Russia \\ c ITMO University, Kronverkskiy pr., 49, 197101 St. Peterssburg, Russia
}

\section{A R T I C L E I N F O}

\section{Article history:}

Received 22 April 2014

Received in revised form

6 August 2014

Accepted 11 August 2014

Available online 20 August 2014

Keywords:

Urine ionic composition

Biochemical analysis of urine

Potentiometric multisensor system

Electronic tongue

Chemometrics

\begin{abstract}
A B S T R A C T
The ionic composition of urine is a good indicator of patient's general condition and allows for diagnostics of certain medical problems such as e.g., urolithiasis. Due to environmental factors and malnutrition the number of registered urinary tract cases continuously increases. Most of the methods currently used for urine analysis are expensive, quite laborious and require skilled personnel. The present work deals with feasibility study of potentiometric multisensor system of 18 ion-selective and cross-sensitive sensors as an analytical tool for determination of urine ionic composition. In total 136 samples from patients of Urolithiasis Laboratory and healthy people were analyzed by the multisensor system as well as by capillary electrophoresis as a reference method. Various chemometric approaches were implemented to relate the data from electrochemical measurements with the reference data. Logistic regression (LR) was applied for classification of samples into healthy and unhealthy producing reasonable misclassification rates. Projection on Latent Structures (PLS) regression was applied for quantitative analysis of ionic composition from potentiometric data. Mean relative errors of simultaneous prediction of sodium, potassium, ammonium, calcium, magnesium, chloride, sulfate, phosphate, urate and creatinine from multisensor system response were in the range 3-13\% for independent test sets. This shows a good promise for development of a fast and inexpensive alternative method for urine analysis.
\end{abstract}

(c) 2014 Elsevier B.V. All rights reserved.

\section{Introduction}

Chemical analysis of biological liquids (blood, saliva, urine, etc.) plays a very significant role in medical diagnostics. Analysis of urine is one of the most informative for medical doctor since it reveals general condition of organism and nutrition character. Urine is an aqueous solution of various substances in different concentrations. It is a convenient object for analysis, components being easily extracted or analyzed right after plain dilution step. Any serious deviation from the normal composition of urine indicates certain metabolic disorder. One of the most widespread metabolic disorders is urolithiasis characterized by the formation of stones in the kidneys and urinary organs [1].

The stone formation begins if equilibrium between urine components is disturbed. The main markers of urolithiasis are separated

\footnotetext{
* Corresponding author at: Chemistry Department, St. Petersburg State University, Universitetskaya nab. 7/9, Mendeleev Center, 199034 St. Petersburg, Russia

E-mail address: d.kirsanov@gmail.com (D. Kirsanov).
}

into promoters and inhibitors. Increased concentrations of promoters (calcium, ammonium, oxalate, phosphate, urate) indicate a risk of possible stone formation. The presence of inhibitors (magnesium, citrate) reduces a possible stone formation since these components enter into competing interaction. The history of urolithiasis markers study is very long [2] and the interest to this issue is growing. The main problem of urolithiasis is that at early stages this disease proceeds asymptomatically. The patient becomes aware of his illness only when the stone is already formed and starts obstructing normal organism activities [3]. Early diagnostics is a half of successful treatment, thus biochemical analysis of urine ionic composition is very important to fight against the urolithiasis. The adequate medical conclusion can be given in laboratory on the basis of biochemical analysis consisted of 14 parameters: contents of sodium, potassium, ammonium, calcium, magnesium, chloride, sulfate, phosphate, oxalate, citrate, urate, creatinine, $\mathrm{pH}$ level and density. Also a registration of sex, age and diuresis is required. The analysis of combination of these parameters can indicate a possible stone formation [4].

Various analytical methods are employed on routine basis for determination of urine composition in medical laboratories. 
Capillary electrophoresis (CE) is considered to be one of the most accurate analytical techniques for urine analysis, offering a number of simple techniques for quantification of inorganic and organic cations and anions in urine. Direct and indirect UVdetection is used most often in CE [4-10]. Spectrophotometric techniques are applied for calcium, magnesium, phosphate, uric acid and creatinine quantification. In this case a preliminary derivatization reaction with specific agent is required to yield colored substance which can be analyzed [11]. Ion-selective electrodes are employed for determination of sodium, potassium and chloride [12].

There are also a number of approaches for urine analysis which were suggested in literature but were not massively introduced into a common laboratory practice so far. A very interesting diagnostic technology is so called "Lithos-system" [13]. The main principle of this system is in observation of crystals of the dried urine sample. The shape and arrangement of crystals is further compared with certain standard figures and this methodology allows to judge on the risk of stone formation [13].

Determination of urine components can be carried out using ion chromatography with piezoelectric or conductivity detectors $[14,15]$. Classical chromatography (liquid and gas) can be successfully applied for analysis of nonionic substances [16-18]. Creatinine and urate as uric acid can be quantified with liquid chromatography-tandem mass spectrometry in urine samples [16,17]. The derivatization with 2,3,4,5,6-pentafluorobenzyl bromide is used for creatinine determination in urine sample by gas chromatography-tandem mass spectrometry [18].

The application of electrochemical methods for urine components analysis is usually hindered by the presence of interfering signals. For example, limited selectivity towards different ions of PVC-plasticized potentiometric sensors in the presence of ammonium lead to attempts to create more selective electrodes for sodium [19] and calcium [20]. An important issue is adsorption of organic urine components on a membrane surface, which leads to reduced reproducibility and lifetime of the sensors. The authors of [21] proposed to use solvent-solvent extraction, membraneimmobilized solvent extraction and solid phase extraction to remove lipophilic compounds from urine samples. However, such kind of sample preparation significantly reduces the benefits of sensors ensuring fast and simple analysis.

There are many reports on application of different biosensors for urine analysis. The analytical signal of these devices is typically based on reactions of target analyte with a biomolecule immobilized on the surface of chemically modified electrode [22]. The content of urate or oxalate in urine can be determined by biosensors based on uricase [23] or oxidase [24] respectively. The main advantage of biosensors is their high selectivity due to specificity of biological reactions. However, it is often hard to obtain reproducible microstructure of a substrate layer to warrant good batch reproducibility.

The idea to use the sensor arrays ("electronic tongues") for simultaneous determination of numerous chemical parameters seems to be quite obvious for urine analysis and there were several attempts reported in literature [25-27]. The authors of [25] have proposed the sensor array consisting of urea biosensor and ionselective electrodes for ammonium, potassium, sodium, hydrogen and one sensor with generic response towards alkaline ions. The response of the array in 37 model solutions was processed with artificial neural network (ANN) and partial least squares (PLS) to construct regression models for simultaneous quantification of urea, ammonium, potassium and sodium. Validation was done with 21 samples (three real urine samples and 18 spiked samples were prepared by addition of different quantities of urea, ammonium, potassium and sodium). It was possible to quantify individual components with relative errors around $8 \%$ for ANN-models and $13 \%$ for PLS-models. Later the same authors extended this sensor array with a creatinine biosensor [26]. TRIS buffer (tris (hydroxymethyl)aminomethane) $\mathrm{pH}=7.5$ was used as a solvent since biosensors require this acidity level to support the catalytic activity of the enzymes. The training of this system was performed on 27 model solutions consisting of five analytes in various concentrations. Independent test-set (13 model solutions) was applied for validation and after that 11 samples (three real urine samples and eight spiked samples were prepared by addition of different quantities of urea, creatinine, ammonium, potassium and sodium) were analyzed. The maximum relative error was $9 \%$ in the determination of creatinine [26].

Another report [27] dealt with the sensor array of eight 8 metallic electrodes (Co, two component alloys - $\mathrm{Sn} / \mathrm{Pb}, \mathrm{Cu} / \mathrm{P}, \mathrm{Cu} / \mathrm{Zn}$, four alloys of silver - $\mathrm{Ag} / \mathrm{Cu} / \mathrm{Zn} / \mathrm{Cd}, \mathrm{Ag} / \mathrm{Cu} / \mathrm{Sn}, \mathrm{Ag} / \mathrm{Cu} / \mathrm{Zn} / \mathrm{Sn}$ - in two different ratios) and five polymeric membrane sensors. The purpose of the study was to detect urinary system dysfunctions and to analyze creatinine levels. 51 urine samples were classified in four classes according to the creatinine content by PLS-Discriminant Analysis (PLS-DA) and neural net. It was shown that this sensor system is able to distinguish between urine samples from healthy patients and those with malignant and non-malignant tumor diagnosis of bladder.

The idea of the present study was to construct the sensor array which would be capable of exhaustive analysis of urine ionic composition. These data are of ultimate importance for early medical diagnosis of several disorders, such as e.g., urolithiasis. Furthermore the intention was to validate this approach with sufficient number of real urine samples employed in independent test sets. The issue of proper validation of promising methods is, unfortunately, ignored in literature pretty often and far-reaching conclusions are being done just with cross-validation on model samples or only a small number of real ones. This is not enough for proper evaluation of such systems for urine analysis.

\section{Materials and methods}

\subsection{Urine samples preparation}

136 urine samples were collected for this study. 117 samples from patients were provided by Urolithiasis Laboratory (Medical Center of Laboratory Diagnostics, St. Petersburg). 19 samples from healthy people were collected from persons who did not express complaints. The accumulation of sufficient number of samples took about six months since only three to six urine samples per week are available from the Urolithiasis Laboratory. All urine samples (50 $\mathrm{ml}$ of each) were frozen in a biomedical fridge at $-25^{\circ} \mathrm{C}$. The literature suggests that ionic composition of urine varies by less than 5 percent on freezing-thawing during 5 years [28].

The urine samples were prepared for analysis with potentiometric multisensor system by unfreezing on a water bath at room temperature $\left(25^{\circ} \mathrm{C}\right)$ and then by thorough mixing. $10 \mathrm{ml}$ of urine were diluted with $90 \mathrm{ml}$ of distilled water and the resulted solution was employed for direct potentiometric measurements. Each sample was measured in 4 physically different replicas. Replicated measurements were randomized between the samples.

\subsection{Potentiometric multisensor systems}

The potentiometric multisensor system consisted of 19 chemical sensors. Ten ion-selective electrodes for $\mathrm{Na}^{+}, \mathrm{K}^{+}, \mathrm{NH}_{4}^{+}, \mathrm{Ca}^{2+}, \mathrm{Mg}^{2+}$, $\mathrm{Ca}^{2+}+\mathrm{Mg}^{2+}, \mathrm{NO}_{3}^{-}, \mathrm{Cl}^{-}, \mathrm{CO}_{3}^{2-}, \mathrm{SO}_{4}^{2-}$ were prepared based on standard Fluka $\AA$ ionophores following the producer recommendations. Five poly (vinylchloride) (PVC)-plasticized anion-sensitive sensors were based on various anion-exchangers, three were PVC-plasticized cation-sensitive 
sensitive sensors based on different tetraphenyl borate derivatives and finally one standard glass $\mathrm{pH}$-electrode. Besides that the system contained the standard $\mathrm{Ag} / \mathrm{AgCl}$ reference electrode. All sensors were connected with shielded wires to the 32-channel high input impedance digital mV-meter (Sensor Systems, LLC, St. Petersburg, Russia). Potential readings were recorded with $0.1 \mathrm{mV}$ precision in a custom made software installed on a Windows PC. Measurement time in each urine sample was $3 \mathrm{~min}$. After that the sensors were washed with three portions of distilled water for $1 \mathrm{~min}$ each. All samples were measured four times. This was done with physically different portions of the same sample. Normally four or five urine samples were defrosted per day and all their replicates were measured in a random order. The results were averaged over the replicas for further processing, the resulted data matrix from the sensor system was 136 samples $\times 19$ sensors.

\subsection{Reference data on urine samples}

All urine samples were analyzed by capillary electrophoresis as a reference method in Bioanalytical Laboratory CSU "Analytical Spectrometry" (St. Petersburg, Russia) according to the procedure described in [4]. Capillary electrophoresis system "Capel $105 \mathrm{M}$ " (Lumex, Russia) with spectrophotometric detector with a wavelength range $190-400 \mathrm{~nm}$ was used for analysis of inorganic cations (calcium, magnesium, sodium, potassium, ammonium) and anions (chloride, sulfate, phosphate) in urine samples diluted in 400-800 times. The content of organic components (oxalate, citrate, urate, creatinine) in 5-20 times diluted urine sample was determined by capillary electrophoresis system Agilent 1100 CE (Agilent, USA) with diode array detector (190-600 nm). pH-level of urine samples was analyzed by pH-sensor InLab Expert Pro (Mettler Toledo, Switzerland). Urine density was evaluated by refractometer IRF-454 B2M (Kazan Optical and Mechanical Plant, Russia).

The data matrix from reference methods was 136 samples $\times$ 14 concentrations.

To yield the medical conclusion on the results diuresis (urine amount excreted per day) has to be considered. For this purpose concentration of particular component in [ $\mathrm{mmol} / \mathrm{l}]$ is multiplied by diuresis in [1/day] to yield the concentration in [mmol/day]. This value is compared with the norm for medical conclusion.

\subsection{Data processing}

Principal Component Analysis (PCA) was employed for exploratory data analysis. PCA is widely in use in modern analytical chemistry and detailed description can be found elsewhere [29]. PCA models were computed with The Unscrambler $\mathbb{R}$ 9.7 (CAMO Software AS, Norway).

Logistic Regression (LR) is a type of probabilistic statistical classification model. It is used for prediction of a categorical dependent variable (i.e. a class label) based on predictor variables (features). In binary Logistic Regression, the outcome is usually encoded as " 0 " or " 1 " as this leads to the most straightforward interpretation. In this study class labels were "unhealthy" or "healthy" and the features were the reference data or the data from the multisensor system. All patients of Urolithiasis Laboratory were considered to be unhealthy. The probability $p(X)$ of a class label is calculated as follows:

$p(X)=\frac{e^{\beta_{0}+\beta_{1} X_{1}+\cdots+\beta_{n} X_{n}}}{1+e^{\beta_{0}+\beta_{1} X_{1}+\cdots+\beta_{n} X_{n}}}$,

where $\beta_{0}, \beta_{1} \ldots \beta_{n}$ are coefficients of the model, $X_{1} \ldots X_{n}$ - predictors (sensor responses or reference data on ionic composition from capillary electrophoresis), and $n$ - number of a predictor.

In assessing statistical medical data it is recommended to consider a prevalence rate for particular location [30]. For this purpose $\beta_{0}$ is transformed in $\beta_{0} *$

$\beta_{0}^{*}=\beta_{0}+\ln \frac{\pi}{1-\pi}-\ln \frac{\pi *}{1-\pi *}$,

where $\pi$ is a morbidity for a certain location (in case of urolithiasis it is up to $15 \%(\pi=0.15)[1])$;

$\pi^{*}$ - a number of the incidence of the disease in the particular study (in our case we have 117 cases seeking medical attention among 136 samples $\left(\pi^{*}=0.86\right)$ ).

LR models were computed with RStudio (Version 0.98.501) and $\mathrm{R}$ (Version 3.0.2) [31].

The quantification of urine components with multisensor system was the main objective of this work. PLS regression was applied for this purpose. Detailed description of PLS regression technique can be found in [29]. The data on ionic composition of urine samples obtained from capillary electrophoresis were employed as reference in modeling. PLS models were computed with The Unscrambler ${ }^{\circledR} 9.7$ (CAMO Software AS, Norway).

\section{Results and discussion}

\subsection{Selecting the optimal sample dilution}

The range of ionic composition of urine in the sample set is known from the reference data. On the other hand, we know the working concentration range of the ion-selective sensors in the array. The comparison shows that for analysis with a multisensor system all the samples can be diluted 5-20 times. It is also noteworthy that dilution can help fighting against the adsorption of organic molecules on polymeric sensor membranes. The series of experiments was performed to select the most appropriate dilution rate. For this purpose urine content in the water was varied over three levels: 5, 10 and 20\%. Urine portions from the same patient were measured five times consequentially at each dilution with multisensor system and sensor readings in these replicas were recorded. Moreover, sensor readings in final washing water between the measurements were also registered. The stability of sensor responses in the water indicates the stability of the sensor array (an imaginary instrument's "baseline").

For each sensor the standard deviation value in five replicated measurements in the urine sample and in five corresponding washing water were calculated. Table 1 shows resulted sums of standard deviations for all of the sensors of the array for three dilution levels.

These results show that with increasing content of the urine the reproducibility of sensor response increases while that in water gets worse. Probably this is associated with higher interference due to more pronounced interaction of organic matter with polymeric sensor membranes at lower dilution rates, which leads to poorer washing. As a compromise between these trends tenfold dilution was chosen for all further experiments.

\subsection{Stability testing of sensors}

All 136 urine samples were analyzed by the multisensor system. It is known [21] that organic components of urine can adsorb on a

\section{Table 1}

The sum of standard deviations of sensor response in replicate measurements at different dilutions levels $(\mathrm{mV})$.

\begin{tabular}{lllr}
\hline & \multicolumn{2}{l}{ Sample dilution } & \\
\cline { 2 - 4 } & $5 \%$ & $\mathbf{1 0 \%}$ & $20 \%$ \\
\hline Sample & 194 & $\mathbf{1 0 9}$ & 79 \\
Water & 112 & $\mathbf{1 2 1}$ & 154 \\
\hline
\end{tabular}




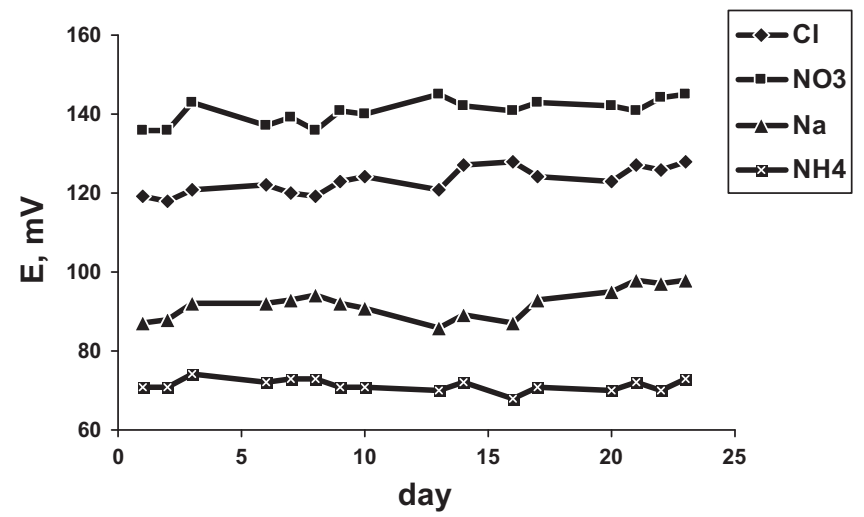

Fig. 1. The responses of four sensors during the experiment.

surface plasticized polymeric membranes causing drift in response values. It was necessary to clarify the impact of this process on the measurements. For this purpose one particular urine sample was split into several portions which were analyzed each day thrice during the whole experiment. The results of these measurements are represented in Fig. 1.

As an example Fig. 1 shows the responses stability of four sensors through 23 days of measurements. For all 19 sensors the standard deviation values of potentials were within $2-10 \mathrm{mV}$, which is probably due to rather high dilution level and extensive washing procedure. This stability was considered to be reasonable for multisensor measurements.

\subsection{Exploratory data analysis}

PCA was applied to get the initial insight into the data. PCA score plots obtained from capillary electrophoresis and multisensor system data (Fig. 2) did not demonstrate the separation between the classes of patients and healthy people. Neither some clustering was visualized in higher PCs. The plots are different and the variance structure is also different for these two methods. The percentage of the explained variance in the first ten PCs was as follows: $39,33,12,6,3,2,2,1,1,1 \%$ - for multisensor system data and $43,14,7,7,6,6,5,5,4,3 \%$ - for data from capillary electrophoresis. The low percentage of the explained variance in the first PCs can be attributed to the fact that there are numerous sources of variance in the data. Thus, the main variability in the data is not associated with a person's status with respect to urolithiasis. This is quite obvious since the urine ionic composition depends on a gender, age, diet and habits. Medical doctors use a complex combination of several parameters for diagnostics. These factors are not highlighted in an overall variance structure and consequently in PCA score plots.

\subsection{Classification of samples}

Logistic Regression models were calculated for two data sets obtained from capillary electrophoresis and potentiometric multisensor system measurements. All samples were split into calibration (101 samples) and validation (35 samples) sets. Probability of class label (unhealthy (1) or healthy (0)) was calculated taking into consideration the incidence of urolithiasis. Prevalence of urolithiasis is up to $15 \%(\pi=0.15)$ [1] while in our data set which was employed for LR calibration there were 87 cases seeking medical attention among 101 samples $(\pi *=0.86)$. The corresponding $\beta_{0}$ values were corrected as described in the experimental section.

The LR models were validated by an independent test-set which contained the data on 30 unhealthy and 5 healthy persons. The results of this analysis are prediction errors in class label
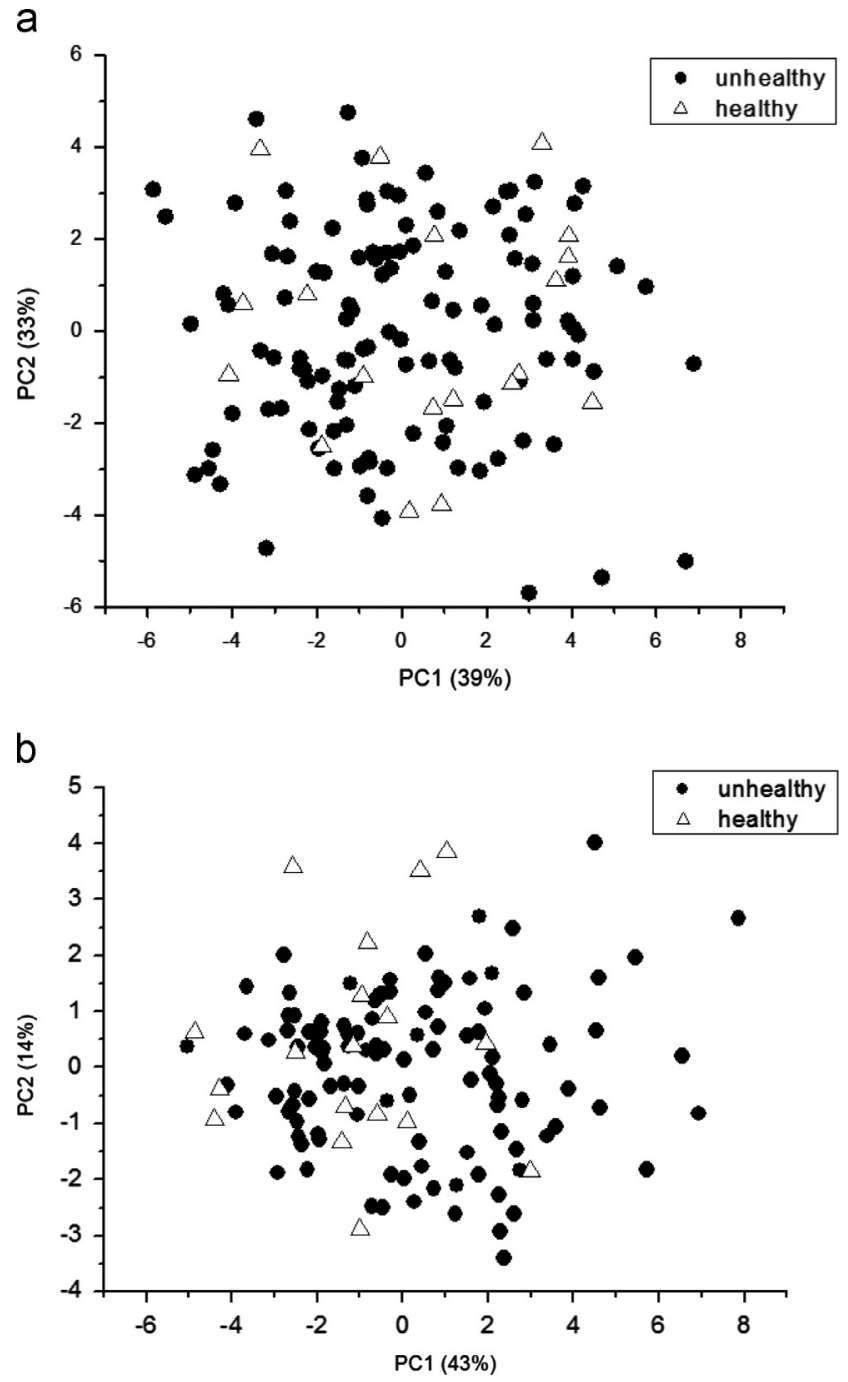

Fig. 2. PCA score plot for multisensor system data (a) and for capillary electrophoresis data (b).

(unhealthy or healthy) from the two data sets. A " 0 " value was assigned to healthy people and " 1 " characterizes the patients of Urolithiasis Laboratory. LR models predict the class label for each sample. The probability $p(X)$ of a class label for each sample has the value between " 0 " and " 1 ". $p(X)$ values which are close to " 1 " assume that there is a high probability of being unhealthy for particular patient.

It is important to establish reliable decision boundary for LR classifier. This boundary determines which particular value of $p(X)$ is used to distinguish between two classes. We have $p(X)$ values of a class label for each sample. Then we choose some particular level $p(X)$ and calculate a number of errors (unhealthy as healthy and healthy as unhealthy) in \%. Then we choose new level $p(X)$ and calculate a number of errors in this case. This operation is repeated with all $p(X)$ values between " 0 " and " 1 ". The way to choose this boundary is to determine $p(X)$. which allows for minimal sum of errors in determination of classes for all samples (Fig. 3). These operations were performed for calibration set only.

Fig. 3 demonstrates minimal sum of errors at decision boundary 0.2 - for capillary electrophoresis data and 0.04 - for multisensor system data.

At these values of decisions boundary the confusion matrix was calculated (Table 2).

It can be seen that LR in case of capillary electrophoresis (CE) allows for $100 \%$ precise classification of the samples, which is not 
a

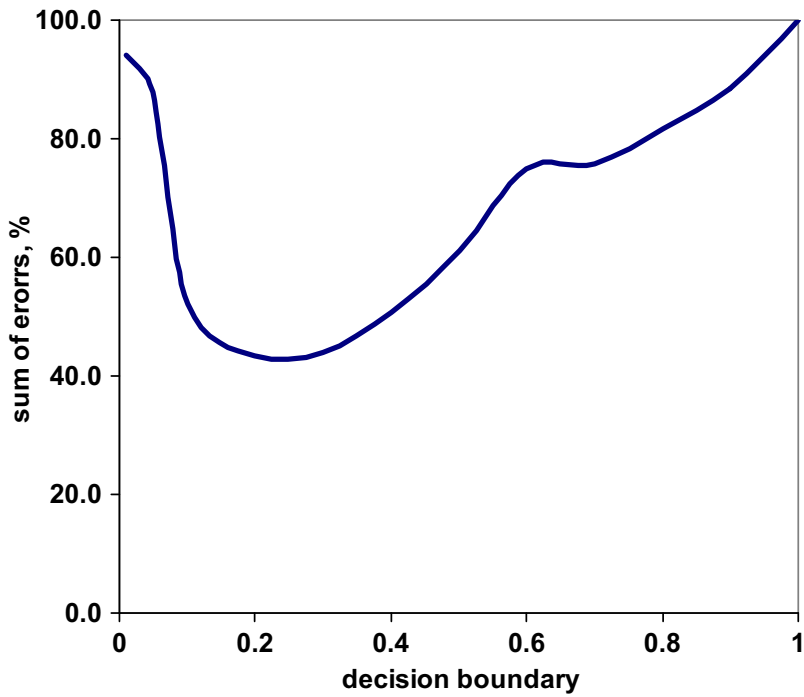

b

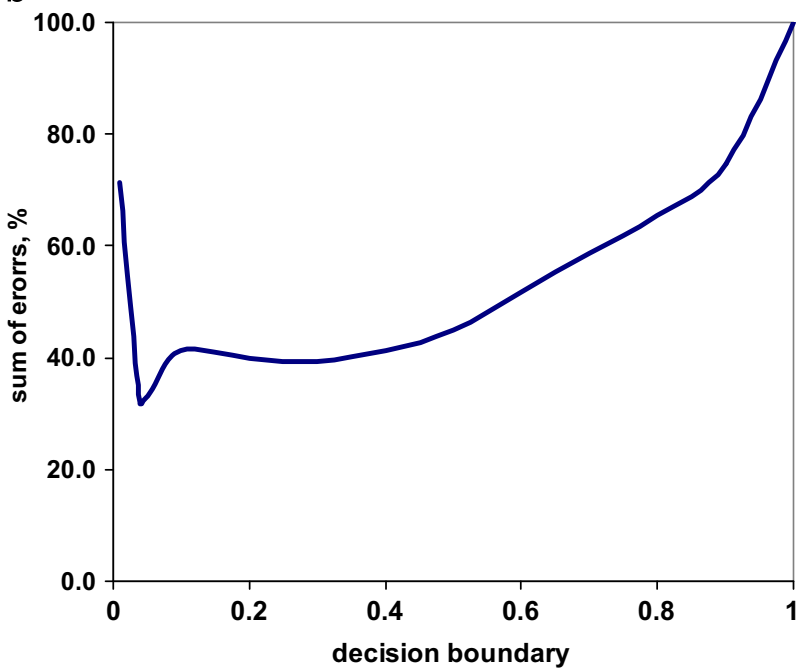

Fig. 3. The choice of decision boundary: (a) - capillary electrophoresis data, and (b) - multisensor system data.

Table 2

Confusion matrix obtained for validation of LR models with independent test set of 35 persons.

\begin{tabular}{lll}
\hline & Unhealthy as healthy & Healthy as unhealthy \\
\hline Capillary electrophoresis data & $0 / 30$ & $0 / 5$ \\
Multisensor system data & $4 / 30$ & $3 / 5$ \\
\hline
\end{tabular}

surprising since these CE data are employed by medical doctors for deriving their conclusions, thus LR in this case just emulates the performance of a medical specialist. Classification precision was somewhat lower when LR models were constructed with potentiometric data, in total 7 out of 35 patients were misclassified.

\subsection{Quantitative analysis}

The quantification of urine components with multisensor system was the main objective of this work. PLS regression models were constructed with reference data from biochemical laboratory. In case of potentiometric sensors there is a linear dependence between sensor response in $\mathrm{mV}$ and logarithm of ion concentration (activity).
Table 3

The mean relative errors in determination of urine compounds (from independent test sets of 40 samples).

\begin{tabular}{lcc}
\hline & Calibration range $(\mathbf{m m o l} / \mathbf{l})$ & MRE $(\%)$ \\
\hline Sodium & $3.8-255.5$ & 2.7 \\
Potassium & $4.2-100.0$ & 8.6 \\
Ammonium & $4.0-81.8$ & 7.2 \\
Calcium & $0.6-10.7$ & 11.6 \\
Magnesium & $0.7-8.7$ & 12.3 \\
Chloride & $11.1-222.3$ & 3.1 \\
Sulfate & $1.5-25.9$ & 10.9 \\
Phosphate & $3.1-48.4$ & 8.2 \\
Oxalate & $0.1-0.4$ & 17.0 \\
Citrate & $0.2-5.5$ & 21.5 \\
Urate & $0.5-7.0$ & 11.5 \\
Creatinine & $2.1-26.7$ & 11.6 \\
\hline
\end{tabular}

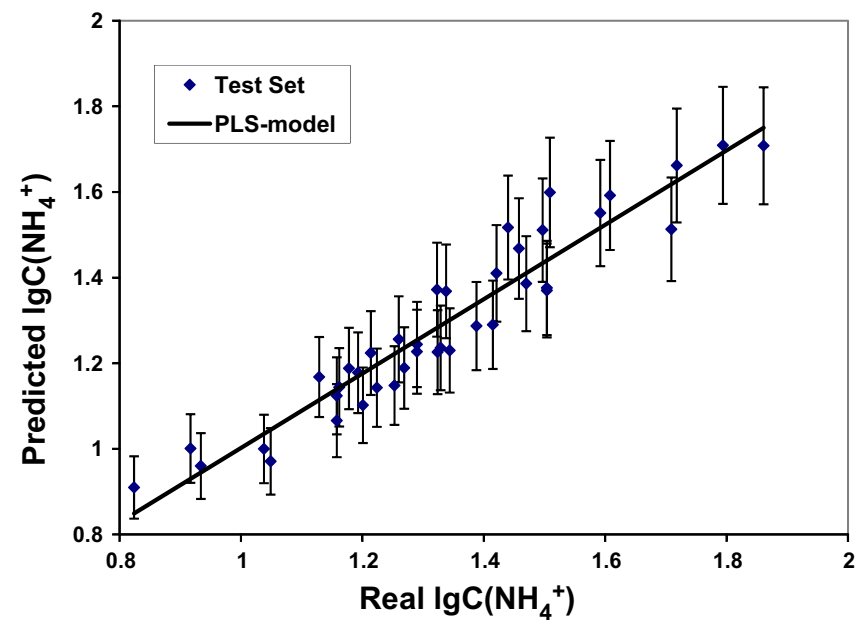

Fig. 4. Measured vs. predicted plot for ammonium.

Thus before modeling the reference data on ion content from capillary electrophoresis were translated into logarithmic scale. Two parameters ( $\mathrm{pH}$ level and density in $\mathrm{g} / \mathrm{l}$ ) were used as is.

40 randomly selected samples were employed as independent test set, while the rest of the samples were employed for building cross-validated PLS1 regression models for each of the 12 parameters. The number of components in PLS models was selected according to traditional criteria - the minimum at the "RMSEP" vs. "number of PLS components" curve. The number of components was typically three (four components were optimal only for ammonium and oxalate). Table 3 represents the results of prediction of these 12 parameters in 40 independent test set samples. Fig. 4 shows typical view of the "measured vs. predicted" plot for ammonium to give an impression on precision of the model at low and high concentrations of analyte.

It was found that potentiometric multisensor system can determine concentrations of certain urine components with mean relative errors in the range $3-13 \%$. The precision in determination of some analytes (oxalate and citrate) was comparatively low. This may be due to the fact that the calibration range for these two ions was quite narrow. Another reason is that significant background concentration of chloride hinders simultaneous determination of small amounts of other ions due to selectivity reasons. Surprising correlation with phosphate content (which is hardly available from potentiometric measurements with polymeric membranes due to its high hydrophilicity) can be partially explained by the fact that this value has reasonably high intercorrelation with all other parameters. For 
a<smiles>CN1CC(=O)[NH2+][C@@H]1C1CC(=O)N([18F])C1</smiles>

b<smiles></smiles>

Fig. 5. The forms of creatinine (a) and uric acid (b) in real urine samples.

example PLS1 regression built with CE data on all ions for prediction of phosphate has $R^{2}$ values around 0.75 in validation.

A high correlation of sensor responses with creatinine and urate contents deserves separate discussion. Creatinine is one of the end products of protein metabolism in human body, its content being important for medical doctors to judge on condition of kidneys and human muscular system. Creatinine is a convenient substance for potentiometric determination because at $\mathrm{pH}$ below 12.3 it exists in a cationic form $\left(\mathrm{p} K_{\mathrm{a}}=12.3\right)$, which can be determined by cation-sensitive sensors. The authors of [27] did manage to achieve RMSEP values for creatinine determination of $0.0019 \mathrm{~mol} / \mathrm{l}$ in model urine samples.

Uric acid and its salts - urates are the end products of purines metabolism. Increased concentrations of urate indicate a possible stone formation. It is dibasic acid $\left(\mathrm{p} K_{\mathrm{a} 1}=5.8, \mathrm{p} K_{\mathrm{a} 2}=10.3\right)$. In urine it forms an anion, which can be analyzed by potentiometric sensors. pH level of urine is normally in the range 5.5-7.0 [1]. The forms of these analytes in real urine samples are shown in Fig. 5. It is assumed that the observed sensitivity of the sensor array towards creatinine and urate levels is associated with these ionic forms; however this issue requires further detailed studies.

In certain cases multisensor system is able to evaluate not only chemical parameters, but also physical. It was possible to determine the density of urine during the analysis. Certainly, the urine density depends on chemical composition. It was established that reference data and multisensor system can help determining density with mean relative errors about $5 \%$ and $8 \%$, respectively, by means of corresponding PLS models with independent test set validation.

\section{Conclusions}

Potentiometric multisensor system can be employed as convenient tool for rapid assessment of 10 ionic substances in human urine (sodium, potassium, ammonium, calcium, magnesium, chloride, sulfate, phosphate, urate, creatinine). The validation of the PLS regression models for quantitative determination of particular constituents with large independent test sets allows for mean relative errors below $10 \%$ for major urine components (sodium, ammonium, chloride) and below 15\% for the rest. The lack of potentiometric selectivity can be effectively compensated in the framework of multisensor approach and chemometric data processing. The further development of this approach into clinical practice looks promising since it is much cheaper and faster than e.g., capillary electrophoretic measurements.

\section{Acknowledgment}

This work was partially financially supported by Government of Russian Federation (Grant 074-U01).

\section{References}

[1] M. Resnick, A. Novick, Urology Secrets, third ed., Hanley and Belfus, Inc., USA, 2003.

[2] G. Gambaro, J.M. Reis-Santos, N. Rao, Eur. Urol. 45 (2004) 547-556.

[2] C. Barbas, A. Garcia, L. Saavedra, M. Muros, J. Chromatogr. B 781 (2002) $433-455$.

[4] A.A. Sidorova, A.V. Grigoriev, J. Anal. Chem. 67 (5) (2012) 478-485.

[5] Q. Wan, P. Kuban, J. Tanyanyiwa, A. Rainelli, P. Hauser, Anal. Chim. Acta 525 (2004) 11-16.

[6] W. Guo, K. Lau, Y. Fung, Electrophoresis 31 (2010) 3044-3052.

[7] V. Galli, A. Garcia, L. Saavedra, C. Barbas, Electrophoresis 24 (2003) 1951-1981.

[8] J. Munoz, M. Lopez-Mesas, M. Valiente, Talanta 81 (2010) 392-397.

[9] A. Garcia, M. Muros, C. Barbas, J. Chromatogr. B 755 (2001) 287-295.

[10] Z. Jarolimova, P. Lubal, V. Kanicky, Talanta 98 (2012) 49-53.

[11] F.T. Fischbach, M.B. Dunning, A Manual of Laboratory and Diagnostic Tests, eighth ed., Lippincott Williams and Wilkins, Philadelphia, 2009.

[12] C.C. Chernecky, B.J. Berger, Laboratory Tests and Diagnostic Procedures, fifth ed., Saunder Elsevier, St. Louis, Missouri, USA, 2008.

[13] V.N. Shabalin, S.N. Shatokhina, Morphology of human biological fluids (Morfologia biologicheskih zhidkostey cheloveka), Chrysostom, Moscow, 2001 (in Russian).

[14] B. Yu, Q. Yuan, L. Nie, S. Yao, J. Pharm. Biomed. Anal. 25 (2001) 1027-1032.

[15] F. Zhao, Z. Wang, H. Wang, R. Zhao, M. Ding, Chin. Chem. Lett. 22 (2011) $342-345$

[16] W. Kwon, J. Kim, S. Suh, M. Kyo M., Forensic Sci. Int. 221 (2012) 57-64.

[17] Y. Zuo, Y. Yang, Z. Zhu, W. Hea, Z. Aydin, Talanta 83 (2011) 1707-1710.

[18] D. Tsikas, A. Wolf, A. Mitschke, F. Gutzki, W. Will, M. Bader, J. Chromatogr. B 878 (2010) 2582-2592.

[19] W. Machini, C. Martin, M. Martinez, S. Teixeira, H. Gomes, M. Teixeira, Sens. Actuators B 181 (2013) 674-680.

[20] S. Beging, D. Mlynek, S. Hataihimakul, A. Poghossian, G. Baldsiefen, H. Busch, N. Laube, L. Kleinen, M. Schoning, Sens. Actuators B, 144 (2010) 374-379.

[21] F. Phillips, K. Kaczor, N. Gandhi, B. Pendley, R. Danish, M. Neuman, B. Toth, V. Horvath, E. Lindner, Talanta 74 (2007) 255-264.

[22] C. Pundir, S. Yadav, A. Kumar, Trends Anal. Chem. 50 (2013) 42-52.

[23] P. Kanyong, R. Pemberton, S. Jackson, J. Hart, Anal. Biochem. 428 (2012) 39-43.

[24] N. Chauhan, J. Narang, A. Shweta, C. Pundir, Clin. Biochem. 45 (2012) 253-258.

[25] M. Gutierrez, S. Alegret, M. Valle, Biosens. Bioelectron. 22 (2007) 2171-2178.

[26] M. Gutierrez, S. Alegret, M. Valle, Biosens. Bioelectron. 23 (2008) 795-802.

[27] L. Lvova, E. Martinelli, F. Dini, A. Bergamini, R. Paolesse, C. Natale, A. Amico, Talanta 77 (2009) 1097-1104.

[28] T. Fenton, M. Eliasziw, A. Lyon, S. Tough, J. Brown, A. David, D. Hanley, Nutr. Res. 29 (2009) 320-326.

[29] K.H. Esbensen, Multivariate Data Analysis - in Practice, CAMO Software, Oslo, 2002.

[30] G. James, D. Witten, T. Hastie, R. Tibshirani, An Introduction to Statistical Learning with Applications in R, Springer Science+Business, Media, New York, 2013.

[31] R Development Core Team. R: A Language and Environment for Statistical Computing, R Foundation for Statistical Computing, Vienna, Austria, 2010 〈http://www.R-project.org $\rangle$. 